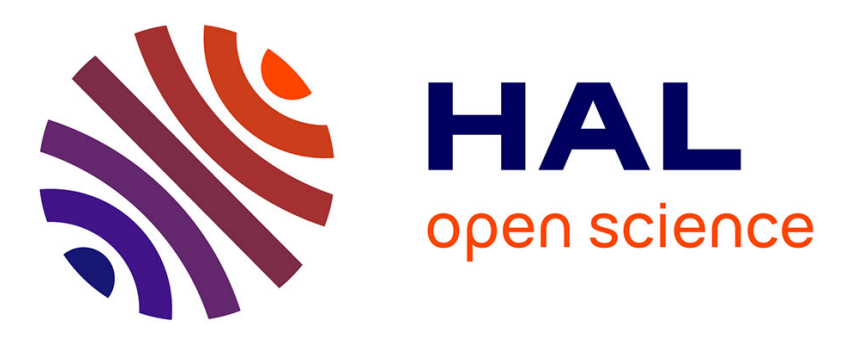

\title{
Road-line detection and 3D reconstruction using fisheye cameras
}

\author{
Rémi Boutteau, Xavier Savatier, Fabien Bonardi, Jean-Yves Ertaud
}

\section{To cite this version:}

Rémi Boutteau, Xavier Savatier, Fabien Bonardi, Jean-Yves Ertaud. Road-line detection and 3D reconstruction using fisheye cameras. 2013 16th International IEEE Conference on Intelligent Transportation Systems - (ITSC 2013), Oct 2013, The Hague, Netherlands. 10.1109/ITSC.2013.6728376 . hal-01710406

\section{HAL Id: hal-01710406 \\ https://hal.science/hal-01710406}

Submitted on 15 Feb 2018

HAL is a multi-disciplinary open access archive for the deposit and dissemination of scientific research documents, whether they are published or not. The documents may come from teaching and research institutions in France or abroad, or from public or private research centers.
L'archive ouverte pluridisciplinaire HAL, est destinée au dépôt et à la diffusion de documents scientifiques de niveau recherche, publiés ou non, émanant des établissements d'enseignement et de recherche français ou étrangers, des laboratoires publics ou privés. 


\title{
Road-line detection and 3D reconstruction using fisheye cameras
}

\author{
R. Boutteau, X. Savatier, F. Bonardi, J.Y. Ertaud \\ Institut de Recherche en Systèmes Electroniques Embarqués (IRSEEM) \\ Technopôle du Madrillet - Avenue Galilée \\ 76800 Saint Etienne du Rouvray - France
}

\begin{abstract}
In future Advanced Driver Assistance Systems (ADAS), smart monitoring of the vehicle environment is a key issue. Fisheye cameras have become popular as they provide a panoramic view with a few low-cost sensors. However, current ADAS systems have limited use as most of the underlying image processing has been designed for perspective views only. In this article we illustrate how the theoretical work done in omnidirectional vision over the past ten years can help to tackle this issue. To do so, we have evaluated a simple algorithm for road line detection based on the unified sphere model in real conditions. We firstly highlight the interest of using fisheye cameras in a vehicle, then we outline our method, we present our experimental results on the detection of lines on a set of 180 images, and finally, we show how the 3D position of the lines can be recovered by triangulation.
\end{abstract}

\section{INTRODUCTION}

In recent years, Advanced Driver Assistance Systems (ADAS), initially limited to luxury vehicles, have become available on high-volume models. Medium-sized cars are now sold with ADAS features such as lane departure warning, blind spot monitoring and road sign recognition. As reported in [1] an impressive growth of the ADAS market is expected, going from $\$ 10$ billion in 2011 to $\$ 130$ billion in 2016. This growth will be possible not only through the reduction of component costs but also with the integration of new functions on the original architecture. This explains why ADAS based on vision are one of the most common schemes for driving assistance as cameras can be considered as versatile multifunction sensors. Recently, [25] has highlighted the performance potential of camera sensor combined with increasingly competitive costs. In the future, as ADAS should offer more and more automated assistance, this will imply a robust and complete perception of the vehicle environment while maintaining affordable costs with sensor rationalization and fusion.

Monitoring the surrounding environment of a car can be achieved using a very small number of cameras. An omnidirectional observation of a scene is possible with a single camera by combining it with a convex mirror. Such a system, called a catadioptric sensor (association of a mirror (catoptric) and lenses (dioptric)), has been widely studied in autonomous robotics [8][33][6]. Its use for automotive applications has also been explored [18][19][27]. However, this sensor is not optimal for the monitoring of the surrounding environment because objects close to the vehicle may be occluded by the vehicle itself. Recently, the use of multiple large field of view cameras has been proposed [10][21]. [29] has demonstrated the potential of such a sensor for pedestrian detection in blind spot monitoring. Indeed, wide angle cameras such as fisheyes are particularly suitable for lateral views as a single camera placed on the side can observe all the surrounding objects from the rear to the front of a car. Thus, only four wide angle cameras are needed to obtain a complete bird's-eye view from above the vehicle as demonstrated with the NISSAN Around view ${ }^{\mathrm{TM}}$ monitor.

Theoretical works in omnidirectional vision have let to the formalization of a unified model which consists of a projection onto a virtual unitary sphere. While initially restricted to central catadioptric sensors [14], this model has recently been extended to fisheye cameras [7]. In past studies, we showed how this framework can be helpful for 3D reconstruction [5] and how it can improve classical machine learning algorithms for image understanding [9]. In this paper, we want to highlight the potential of such a model coupled with fisheye sensors for an ADAS application by applying it to roadmarking detection. Road-marking detection is a basic step in many ADAS functions such as lane-departure warning, lanekeeping and blind-spot control. In this paper, we present a simple and efficient algorithm for detecting lane marking using lateral fisheye views. As a first step, the algorithm is restricted to straight line detection and is evaluated in real conditions on a database of 180 images. Using our method, a $3 \mathrm{D}$ reconstruction of lines is possible, which facilitates the rejection of outliers and will be helpful in automated guidance. In section II, we set out the state of the art with regard to road-line detection algorithms in classical and omnidirectional images. In section III, we present our new algorithm for detecting straight lines in omnidirectional images. Section IV is dedicated to the experimental results obtained from this detection algorithm as well as to a straight line triangulation application. Lastly, in section V, we present a conclusion and identify several directions for future work.

\section{STATE OF THE ART IN METHODS FOR DETECTING STRAIGHT LINES IN OMNIDIRECTIONAL IMAGES}

Road-marking detection for ADAS applications has been widely studied using monocular systems [22], stereovision [24] or more recently by fusion with navigation sensors [28] but most of the algorithms proposed are suitable for perspective front views only. An interesting survey of common algorithms can be found in [32]. Lane detection 
obtained from lateral views is, however, particularly suitable in applications where lateral parts of the vehicle have to be monitored. But in such a case, perspective cameras cannot be used due to the fact that surrounding objects may be very close to the car. In [20], the authors demonstrated how this issue can be tackled using fisheye cameras. They proposed a method to detect road marking based on some assumptions regarding the movement of cars. In [21] they also used their line detection method for on-line fisheye calibration. Another interesting use of fisheyes can be found in [16] where the authors used this sensor for car recognition.

The detection of straight lines in omnidirectional images is much less easy than in perspective images as the projection of lines onto an image plane no longer gives straight lines but conic sections. The classical problem of detecting lines in perspective images therefore becomes a complex problem of detecting conics in omnidirectional images. There are several special cases which simplify the detection of lines in omnidirectional images. [34] and [8] were interested in the detection of lines projected radially onto a conical mirror. This configuration requires that the sensor be in a vertical position and that there be sufficient vertical lines in the environment. [12] proposed a method for extracting horizontal and vertical segments but not for every type of straight line. This limitation is due to the use of non-central sensors which makes the detection of certain types of lines extremely complex. The same problem is covered in [26]. For central sensors, the methods for estimating lines can be classified into two main categories. The first category tries to detect conic sections in the image and to estimate the five parameters so that the conic section is adjusted to the extracted data [36]. The computation time is generally long and these algorithms are not efficient when only small proportions of the conic sections are visible. Furthermore, these methods are often only applicable in the special case of the paracatadioptric sensor, a combination of a paraboloidal mirror and an orthographic camera, as the projections of the lines then become arcs of circles [2] [3] [30]. The second category involves the detection of great circles on the equivalence sphere. It has been demonstrated in [15] that the projection of a 3D line on the sphere is a great circle $C$ as illustrated in Figure 1. These methods are only applicable to calibrated sensors as it is necessary to be able to calculate the projection of pixels on the equivalence sphere. [4] describes such a method.

The main advantage of the detection of great circles on the equivalence sphere is that the algorithm can be used for all central sensors because it is a unified model. The detection of great circles is in general carried out by a Hough transformation [17], the difference between the various algorithms is the space in which the processing is carried out. In [31], the great circles on the sphere are defined by their normal, characterized by the angles of elevation and azimuth. A Hough space based on these angles is then created and the detection of maxima in the Hough space enables the parameters for the lines to be obtained. There are other Hough spaces for characterizing the lines. It is for

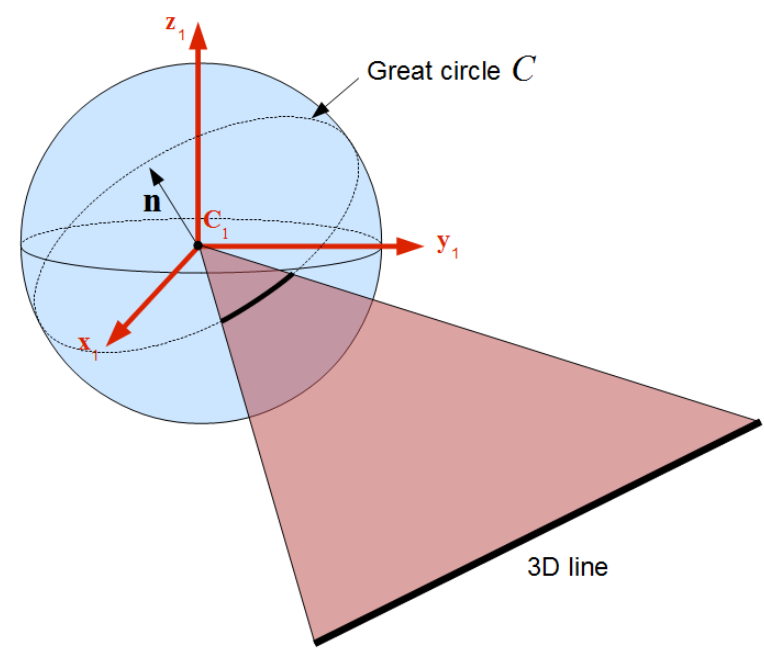

Fig. 1. Projection of a line onto the equivalence sphere.

example possible to use either the normal directly [23] or its cubic coordinates [35]. However, the Hough transformation introduces several problems such as the selection of the best sampling step for the Hough space which should in addition take account of the non-uniform resolution of the sensors. In addition, the detection of maxima in the Hough space is a complicated step.

\section{OUR METHOD}

As we have seen in the previous section, most of the existing algorithms are either specific to a type of mirror or specific to a given type of line (horizontal or vertical). We therefore propose a new method for detecting and estimating any type of line in omnidirectional images, valid for all central sensors and based on the robust RANSAC (RANdom SAmple Consensus [13]) estimation algorithm. The first phase in our method consists in extracting the edges present in the omnidirectional image (figure 2a) by applying Canny's algorithm. A very large number of edges are detected some of which are not actually physical edges, as is shown in Figure 2b. Chains of connected pixels are then created with the edges previously extracted. To avoid detecting a multitude of small lines, chains which are too short are disregarded. The result of this step is shown in Figure 2c. In this figure, the chains of connected pixels are represented by different colors and chains fewer than 50 pixels have been discarded.
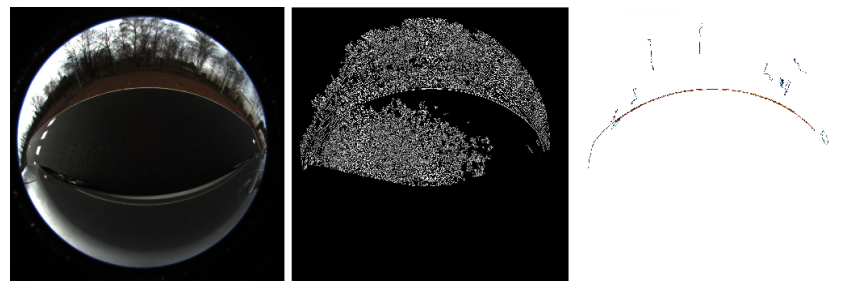

Fig. 2. (a) Omnidirectional image - (b) Detected edges- (c) Chains of connected pixels.

Each chain thus created is a potential omnidirectional line. 
It is therefore necessary to make a decision on whether it really is a line and, if so, estimate its parameters. Omnidirectional lines will be characterized by the normal to the plane which contains the line and which passes through the center of the equivalence sphere (see Figure 3).

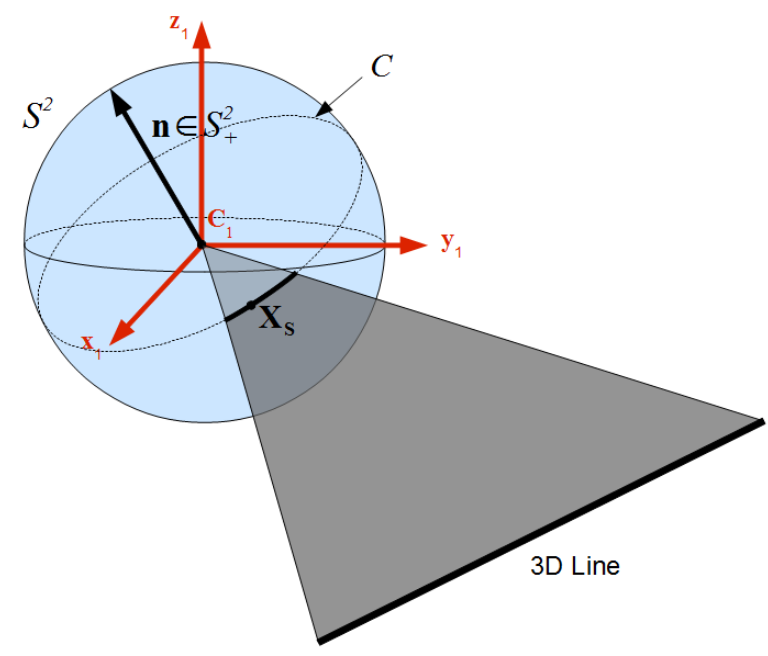

Fig. 3. Characterisation of an omnidirectional line by a normal.

We propose to work directly on the unitary sphere $S^{2} \in$ $\mathbb{R}^{3}$. We characterize a line by the normal $\mathbf{n} \in S_{+}^{2}$ to the plane formed by the line and the center of projection. All the pixels in the chains are projected onto the equivalence sphere. This step, called lifting, requires knowledge of the intrinsic parameters of the sensor and therefore assumes the sensor to be calibrated. All the following calculations are carried out using the points on the sphere. To estimate the parameters of the line which best correspond to the points in the chain, a robust estimation method called RANSAC is used. Its pseudo-code is given in Algorithm 1. For each chain, two points $\mathbf{X}_{\mathbf{S}}^{\mathbf{1}}$ and $\mathbf{X}_{\mathbf{S}}^{2}$ are taken randomly and the normal to the plane passing through these two points and the center of the sphere is calculated:

$\mathbf{n}=\lambda \frac{\mathbf{X}_{\mathbf{S}}^{1} \times \mathbf{X}_{\mathbf{S}}^{2}}{\left\|\mathbf{X}_{\mathbf{S}}^{1} \times \mathbf{X}_{\mathbf{S}}^{2}\right\|}$, where $\lambda \in\{-1,1\}$ ensures that $\mathbf{n} \in S_{+}^{2}$

The other points in the chain are then tested to decide whether they belong to the great circle $C$ given by:

$$
C=\left\{\mathbf{X}_{\mathbf{S}} \mid \mathbf{n}^{T} \mathbf{X}_{\mathbf{S}}=0,\left\|\mathbf{X}_{\mathbf{S}}\right\|=1\right\}
$$

In practice, because there are small errors due to noise, the discretization of the image or calibration, an error threshold is set to decide whether or not point $\mathbf{X}_{\mathbf{S}}$ belongs to the great circle $C$. All the points belonging to the great circle $C$ defined by $\mathbf{n}$ are then stored in the memory. If the number of points belonging to the circle $C$ is greater than the minimum length allowed for defining a line, then the pixel chain does indeed relate to a line whose normal is re-estimated using all the points belonging to the great circle. It is then possible to define segments rather than lines by taking the first and last pixel in the chain belonging to the great circle.

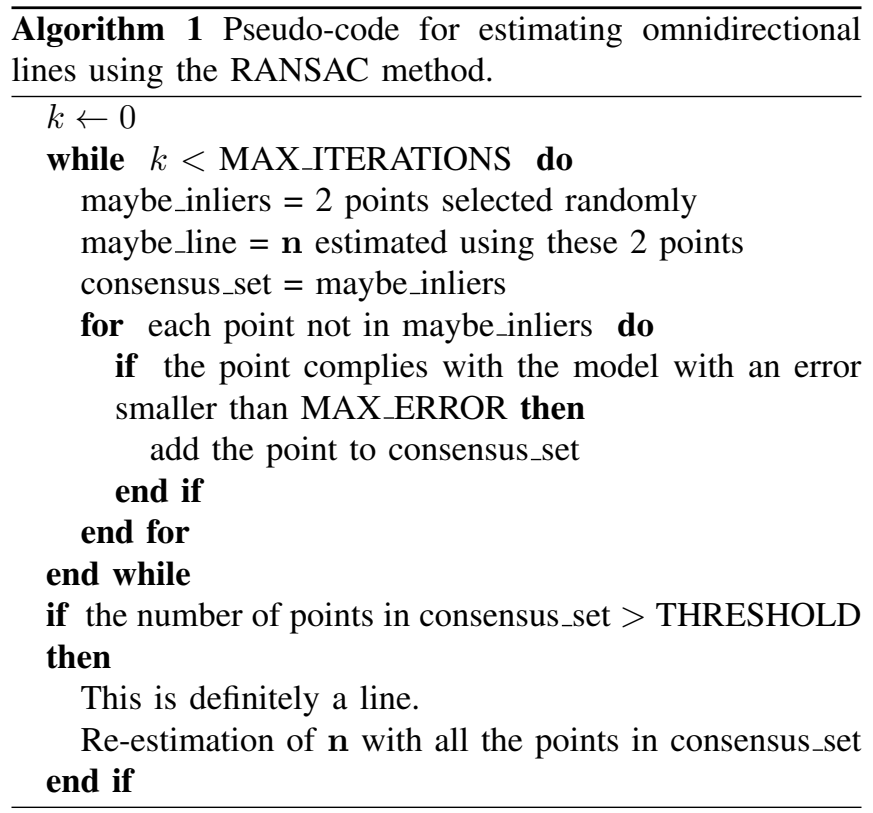

\section{EXPERIMENTAL RESULTS AND APPLICATION TO LINE TRIANGULATION}

\section{A. Line detection}

We applied this algorithm to the field of autonomous computer-aided driving, so we chose to place our cameras close to the left and right rear-view mirrors of a car. The camera we used is a Prosilica GC1380C with a fisheye lens (Fujinon fisheye 1:1.4/1.8mm) reducing its region of interest to a resolution of $1024 \times 1024$ pixels so that the circular field of view is centered in the images. We pointed this sensor toward the road with an angle of about $45^{\circ}$ in order to see both the immediate environment (road and blind spots of the car) and the global environment (buildings for instance). The sensor was calibrated using our omnidirectional calibration tool ${ }^{1}$. During testing we drove both in urban zones and on highways, with several quality levels of the road (texture, uniformity, etc) and its markings (good, damaged or missing). We took 180 images, which were later processed with our algorithm. To evaluate the sensitivity of the processing, we calculated the True Positive Rate (TPR) according to the following formula:

$$
T P R=\frac{T P}{T P+F N}
$$

- True Positives (TP) are cases where there is a road marking or a border of the road in the image and the algorithm detects it (see Figure 4a and 4b).

- False Negatives (FN) are cases where there is a line or a border of the road in the image and the algorithm does not detect it (see Figure 4d).

\footnotetext{
${ }^{1}$ This software is available at http://omni3d.esigelec.fr/doku.php/omni/calib
} 
- False Positives (FP) are cases where the algorithm detects a line where there is none (see Figure 4c).

- During our tests, we did not encounter any True Negatives (TN), due to the fact that there was always a road marking or a border of the road in the field of view.

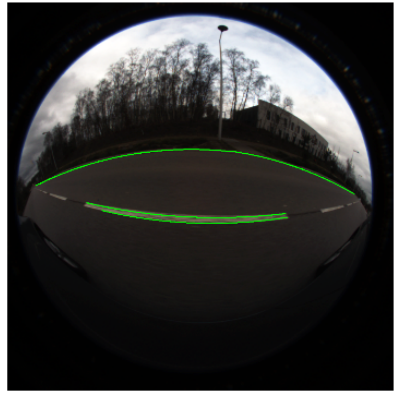

(a)

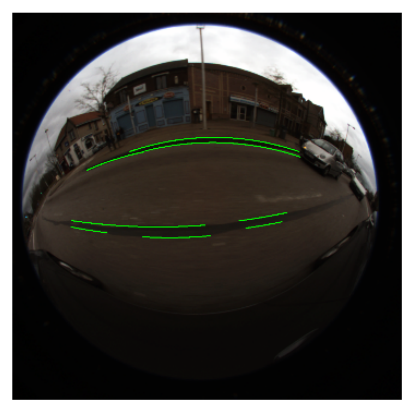

(c)

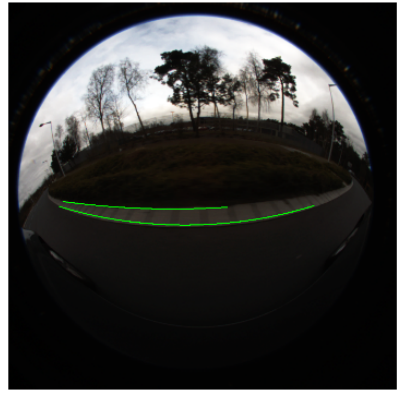

(b)

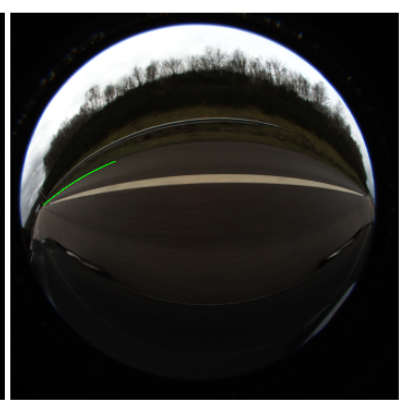

(d)
Fig. 4. Samples of line detection. (a) and (b) True Positives, (c) False Positive, (d) False Negative

The TP, FN and FP rates are given in Table I.As shown, we obtained a True Positive Rate of $86.9 \%$.

TABLE I

DETECTION RATES OBTAINED ON THE 180 IMAGES OF THE DATABASE

\begin{tabular}{|c|c|}
\hline TP & $81.11 \%$ \\
\hline FN & $12.22 \%$ \\
\hline FP & $6.67 \%$ \\
\hline TPR & $86.9 \%$ \\
\hline
\end{tabular}

\section{B. $3 D$ reconstruction}

The detection of omnidirectional lines outlined above enables us to parameterize the line in the local reference frame of the sensor, the line being represented by the normal to the plane containing the line and the center of the sensor. It is possible to triangulate the line, in order to obtain its 3D position. This can be achieved if the pose $(\mathbf{R}, \mathbf{t})$ of the sensor relative to the road is known. In practice, we used a calibration pattern lying on the road to estimate the pose by solving an omnidirectional Perspective-N-Point problem [11].

Let $\left(\mathbf{C}_{1}, \mathbf{x}_{1}, \mathbf{y}_{1}, \mathbf{z}_{1}\right)$ be the reference frame attached to the vehicle (see Figure 5). The center $\mathbf{C}_{\mathbf{1}}=\left[\begin{array}{lll}0 & 0 & 0\end{array}\right]^{T}$ is located vertically below the omnidirectional sensor and on the road level. $\mathbf{n}_{\mathbf{1}}=\left[\begin{array}{lll}0 & 0 & 1\end{array}\right]^{T}$ is the normal of the road.
Let $\mathbf{C}_{\mathbf{2}}=\left[\begin{array}{lll}c_{2 x} & c_{2 y} & c_{2 z}\end{array}\right]^{T}$ be the center of the sensor, and $\left(\mathbf{C}_{2}, \mathbf{x}_{2}, \mathbf{y}_{2}, \mathbf{z}_{2}\right)$ the reference frame of this sensor. $\mathbf{n}_{2}=$ $\left[\begin{array}{lll}n_{2 x} & n_{2 y} & n_{2 z}\end{array}\right]^{T}$ is the normal characterizing the line within the reference frame $\left(\mathbf{C}_{\mathbf{1}}, \mathbf{x}_{\mathbf{1}}, \mathbf{y}_{\mathbf{1}}, \mathbf{z}_{\mathbf{1}}\right)$. The objective is to determine the parametric equation of the corresponding $3 \mathrm{D}$ line $L: L=\mathbf{A}+\lambda \mathbf{u}$, where $\mathbf{A}$ is a point on the line and $\mathbf{u}$ its direction vector.

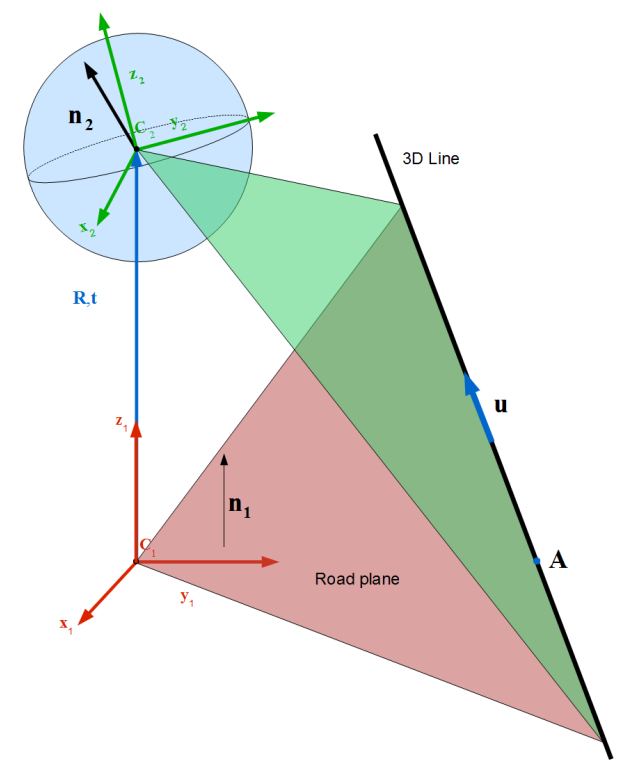

Fig. 5. Triangulation of an omnidirectional line.

The line verifies the equation of the two planes, therefore:

$$
\left\{\begin{aligned}
z & =0 \\
n_{2 x} \cdot x+n_{2 y} \cdot y+n_{2 z} \cdot z-n_{2 z} \cdot c_{2 z} & =0
\end{aligned}\right.
$$

In $z=0$ and $x=0$, line $L$ therefore passes through point $\mathbf{A}$ with coordinates $\mathbf{A}=\left[0 \frac{n_{2 z} \cdot c_{2 z}}{n_{2 y}} 0\right]^{T}$.

The direction vector $\mathbf{u}$ of the line is obtained directly by calculating the cross product of the normals of the two planes defining this line:

$$
\mathbf{u}=\frac{\mathbf{n}_{\mathbf{1}} \times \mathbf{n}_{\mathbf{2}}}{\left\|\mathbf{n}_{\mathbf{1}} \times \mathbf{n}_{\mathbf{2}}\right\|}
$$

The 3D estimation of the line was evaluated in a corridor as shown in Figure 6. The sensor was mounted on a robot and calibrated as described above.

The line corresponding to the bottom of the wall was detected and its 3D position was compared with the ground truth obtained using a 3D laser scanner (Leica ScanStation C10). Figure 7 shows that the estimated position of the line (in green) corresponds to the ground truth obtained by laser telemetry (in gray).

\section{CONCLUSION AND FUTURE WORK}

In this paper, we have presented a novel method for line detection in omnidirectional images which is able to extract any straight lines (not only vertical ones) with any central sensor (fisheye, or catadioptric sensors of any shape). We 


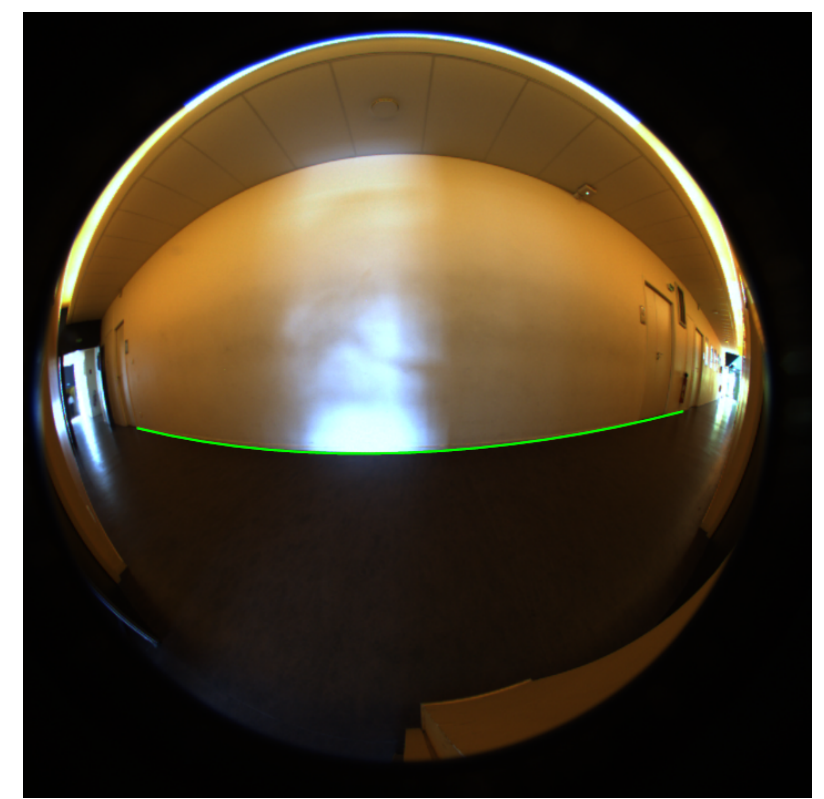

Fig. 6. The omnidirectional image used for the 3D reconstruction of the line.

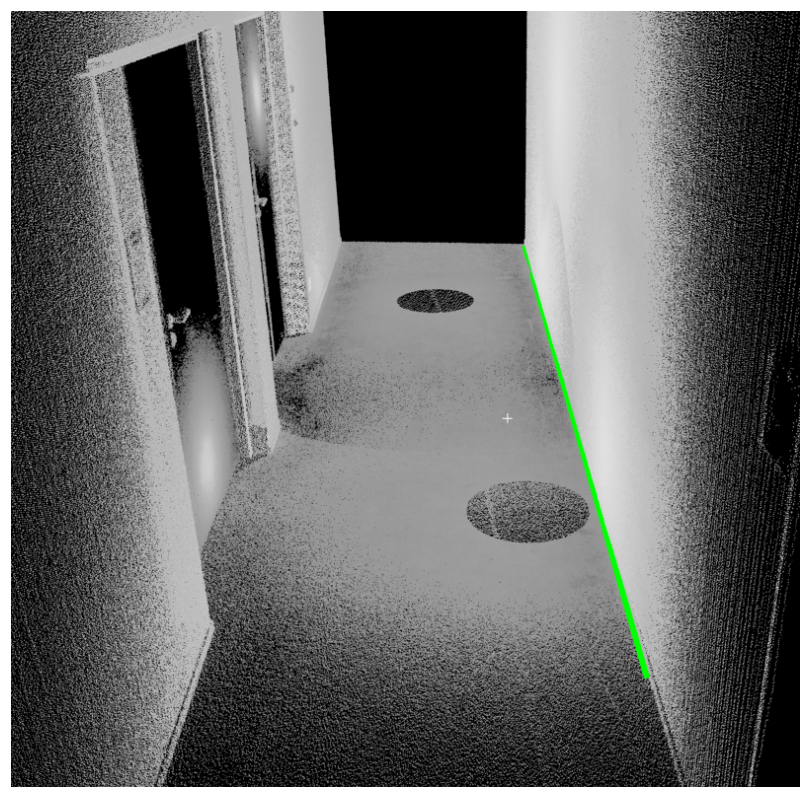

Fig. 7. 3D reconstruction of a corridor. The triangulated line is represented in green and the ground truth in gray.

applied our algorithm to road line detection with fisheye cameras embedded in a vehicle. The True Positive Rate of $86.9 \%$ that we obtained demonstrates that the proposed technique works very well. It is also possible to obtain the 3D position of the detected lines, using triangulation, which can be useful for ADAS features such as lane departure warning or for the visual servoing of vehicles on the road. Our future works will focus on the problem of tracking the lines over time to improve the results in terms of robustness.

\section{ACKNOWLEDGMENT}

This study has been supported by the European ARTEMIS Joint Undertaking and the DGCIS at the French Ministry for Industrial Renewal.

\section{REFERENCES}

[1] Advanced driver assistance systems (adas). Technical report, ABIresearch, 2011.

[2] J.P. Barreto and H. Araujo. Direct least square fitting of paracatadioptric line images. In IEEE Conference on Computer Vision and Pattern Recognition Workshop (CVPRW), volume 7, pages 78-84, 2003.

[3] J.P. Barreto and H. Araujo. Fitting conics to paracatadioptric projections of lines. Computer Vision and Image Understanding (CVIU), 101(3):151-165, 2006.

[4] J.C. Bazin, C. Demonceaux, and P. Vasseur. Fast central catadioptric line extraction. In Iberian Conference on Pattern Recognition and Image Analysis (IbPRIA), volume 4478, pages 25-32, 2007.

[5] R. Boutteau. 3d reconstruction for autonomous navigation. http://omni3d.esigelec.fr/doku.php/thesis/r3d/start, 2009.

[6] R. Boutteau, X. Savatier, J.Y. Ertaud, and B. Mazari. An omnidirectional stereoscopic system for mobile robot navigation. Sensors \& Transducers Journal, Special Issue on Robotic and Sensors Environments, 5:3-17, March 2009.

[7] Jonathan Courbon, Youcef Mezouar, Laurent Eck, and Philippe Martinet. A generic fisheye camera model for robotic applications. In Intelligent Robots and Systems, 2007. IROS 2007. IEEE/RSJ International Conference on, pages 1683-1688. IEEE, 2007.

[8] L. Delahoche, C. Pgard, B. Marhic, and P. Vasseur. A navigation system based on an omnidirectional vision sensor. In IEEE/RSJ International Conference on Intelligent Robots and Systems (IROS), volume 2, pages 718-724, 1997.

[9] Y. Dupuis, X. Savatier, J. Ertaud, and P. Vasseur. Robust radial face detection for omnidirectional vision. Image Processing, IEEE Transactions on, PP(99):1, 2012.

[10] Tobias Ehlgen, Tomáš Pajdla, and Dieter Ammon. Eliminating blind spots for assisted driving. Intelligent Transportation Systems, IEEE Transactions on, 9(4):657-665, 2008.

[11] J. Fabrizio and J. Devars. An analytical solution to the perspective-npoint problem for common planar camera and for catadioptric sensor. International Journal of Image and Graphics (IJIG), 8(1):135-155, 2008.

[12] M. Fiala and A. Basu. Line segment extraction in panoramic images In International Conference in Central Europe on Computer Graphics, Visualization and Computer Vision (WSCG), pages 179-186, 2002.

[13] M.A. Fischler and R.C. Bolles. Random sample consensus: A paradigm for model fitting with applications to image analysis and automated cartography. In Communications of the ACM, volume 24, pages 381-395, June 1981.

[14] C. Geyer and K. Daniilidis. A unifying theory for central panoramic systems and practical implications. In European Conference on Computer Vision (ECCV), pages 445-461, Dublin, Ireland, June 2000. Springer, Berlin.

[15] C. Geyer and K. Daniilidis. Catadioptric projective geometry. International Journal of Computer Vision (IJCV), 43(3):223-243, 2001.

[16] Kenichi Hirose, Takashi Toriu, and Hiromitsu Hama. Estimation of vehicle wheelbase in a circular fisheye image using two-step detection method of tire-road contact points. Int J Innov Comput Inf Control, 7(8):4717-4728, 2011.

[17] P.V.C. Hough. Method and means for recognizing complex patterns. U.S. Patent 3069654, December 1962.

[18] Kohsia S Huang, Mohan M Trivedi, and Tarak Gandhi. Driver's view and vehicle surround estimation using omnidirectional video stream. In Intelligent Vehicles Symposium, 2003. Proceedings. IEEE, pages 444-449. IEEE, 2003.

[19] J.F. Layerle, , X. Savatier, J.Y. Ertaud, and E.M. Mouaddib. Catadioptric sensor for a simultaneous tracking of the driver's face and the road scene. In Workshop on Omnidirectional Vision, Camera Networks and Non-classical Cameras (OMNIVIS), 2008.

[20] Shigang Li. Monitoring around a vehicle by a spherical image sensor. Intelligent Transportation Systems, IEEE Transactions on, 7(4):541$550,2006$. 
[21] Shigang $\mathrm{Li}$ and Ying Hai. Easy calibration of a blind-spot-free fisheye camera system using a scene of a parking space. Intelligent Transportation Systems, IEEE Transactions on, 12(1):232-242, 2011.

[22] J.C. McCall and M.M. Trivedi. Video-based lane estimation and tracking for driver assistance: survey, system, and evaluation. Intelligent Transportation Systems, IEEE Transactions on, 7(1):20-37, 2006.

[23] C. Mei. Laser-Augmented Omnidirectional Vision for 3D Localisation and Mapping. PhD thesis, Mines de Paris, February 2007.

[24] Sergiu Nedevschi, Rolf Schmidt, Thorsten Graf, Radu Danescu, Dan Frentiu, Tiberiu Marita, Florin Oniga, and Ciprian Pocol. 3d lane detection system based on stereovision. In Intelligent Transportation Systems, 2004. Proceedings. The 7th International IEEE Conference on, pages 161-166. IEEE, 2004.

[25] M. Pajon, S. Cornou, E. Debernard, Ph. Mathieu, J. Mousain, Y. Page, and D. Wautier. Enlighting the future: from autonomous adas to autonomous driving. In Vehicle and Infrastructure Safety Improvement in Adverse Conditions and Night Driving (VISION) 2012, 2012.

[26] R. Pinciroli, A. Bonarini, and M. Matteucci. Robust detection of $3 \mathrm{~d}$ scene horizontal and vertical lines in conical catadioptric sensors. In Workshop on Omnidirectional Vision, Camera Networks and Nonclassical Cameras (OMNIVIS), 2005.

[27] D. Scaramuzza. 1-point-ransac structure from motion for vehiclemounted cameras by exploiting non-holonomic constraints. International Journal of Computer Vision (IJCV), 95(1):74-85, 2011.

[28] S. Sivaraman and M.M. Trivedi. Improved vision-based lane tracker performance using vehicle localization. In Intelligent Vehicles Symposium (IV), 2010 IEEE, pages 676-681, 2010.

[29] H.H. Tadjine, B. Auerbach, and K. Karsten. Intelligent park assists system: Pedestrian detection based on fisheye camera. In Vehicle and Infrastructure Safety Improvement in Adverse Conditions and Night Driving (VISION), 2012.

[30] B. Vandeportaele, M. Cattoen, and P. Marthon. A fast detector of line images acquired by an uncalibrated paracatadioptric camera. In International Conference on Pattern Recognition (ICPR), pages 10421045, 2006.

[31] P. Vasseur and E.M. Mouaddib. Central catadioptric line extraction. In British Machine Vision Conference (BMVC), pages 57-66, 2004.

[32] T. Veit, J. P Tarel, P. Nicolle, and P. Charbonnier. Evaluation of road marking feature extraction. In Intelligent Transportation Systems, 2008. ITSC 2008. 11th International IEEE Conference on, pages 174181, 2008.

[33] Y. Yagi. Omnidirectional sensing and its applications. IEICE Transactions on Information and Systems, E82-D(3):568-579, March 1999.

[34] Y. Yagi and M. Yachida. Real-time generation of environmental map and obstacle avoidance using omnidirectional image sensor with conic mirror. In IEEE Conference on Computer Vision and Pattern Recognition (CVPR), pages 160-165, 1991.

[35] K. Yamazawa, Y. Yagi, and M. Yachida. 3d line segment reconstruction by using hyperomni vision andomnidirectional hough transforming. In International Conference on Pattern Recognition (ICPR), volume 3, pages 483-486, 2000.

[36] Z. Zhang. Parameter estimation techniques: A tutorial with application to conic fitting. Image and Vision Computing Journal, 15(1):59-76, 1997. 